\title{
Giant cell lichenoid stomatitis - An oral medicine case book
}

SADJ November 2020, Vol. 75 No. 10 p550 - p552

\author{
L Robinson', L Kotze ${ }^{2}$, WFP van Heerden ${ }^{3}$
}

\section{CASE REPORT}

A 53-year-old female patient presented with a two-week history of intermittently painful oral mucosal lesions when eating spicy, salty or sour foods. The patient reported a long-term history of psoriasis treated by topical corticosteroids only. The remainder of the patient's medical history was unremarkable. On intraoral examination, lesions were noted bilaterally in the posterior buccal commissures, appearing as centrally erosive lesions bound by white striations (Figure 1). Additionally, there were areas of desquamative gingivitis in the anterior maxillary gingiva. The clinical suspicion was that of oral lichen planus or oral lichenoid lesions. An incision biopsy from the left buccal mucosa was performed and submitted for histological assessment.
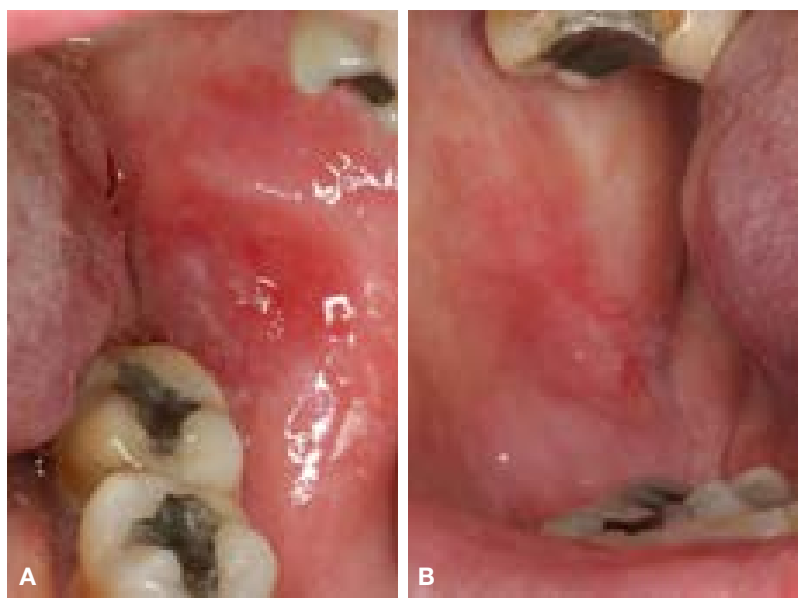

Figure 1. Initial clinical presentation: Left (A) and right (B) buccal mucosae with erosive lesions bound by white striae.

\section{Author affiliations:}

1. Liam Robinson: $B C h D, P D D$ (Maxillofacial Radiology), PDD (Forensic Odontology), Department of Oral Pathology and Oral Biology, School of Dentistry, Faculty of Health Sciences, University of Pretoria, South Africa.

ORCID Number: 0000-0002-0549-7824

2. Leande Kotze: $B C h D$, Department of Periodontics and Oral Medicine, School of Dentistry, Faculty of Health Sciences, University of Pretoria.

ORCID Number: 0000-0003-1518-4941

3. Willie FP van Heerden: BChD, MChD, FC Path (SA) Oral Path, $P h D, D S c$, Department of Oral Pathology and Oral Biology, School of Dentistry, Faculty of Health Sciences, University of Pretoria, South Africa.

ORCID Number: 0000-0003-2494-667X

\section{Corresponding author: Willie FP van Heerden}

Department of Oral Pathology and Oral Biology, University of Pretoria, South Africa.

Email: willie.vanheerden@up.ac.za

Author contributions:

1. Liam Robinson: Principle author $-40 \%$

2. Leande Kotze: Clinical case and treatment $-20 \%$

3. Willie FP van Heerden: Diagnosis, histological images, advisor $-40 \%$

\section{Pathologic findings}

The specimen consisted of an elliptical mucosal biopsy measuring $4 \times 4 \times 2 \mathrm{~mm}$. Histological evaluation showed a tissue fragment surfaced by atrophic parakeratinising stratified squamous epithelium. The basal cell layer showed areas of vacuolar degeneration with focal Civatte and colloid bodies.

The lamina propria revealed a dense band-like inflammatory cell infiltrate with isolated lymphoid follicles, some with activated germinal centres (Figure 2). This infiltrate consisted of a mixed population of inflammatory cells including lymphocytes, histiocytes and scattered plasma cells. Of note, the infiltrate contained an unusual population of multinucleated giant cells (Figure 3).

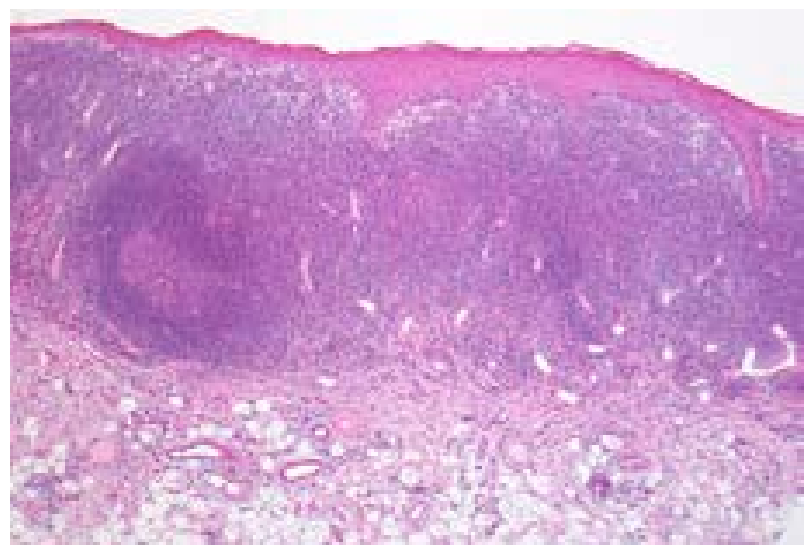

Figure 2. Low-power hematoxylin and eosin (H\&E)-stained section showing the dense lichenoid inflammatory cell infiltrate with isolated lymphoid follicles (original magnification $x 40$ ).

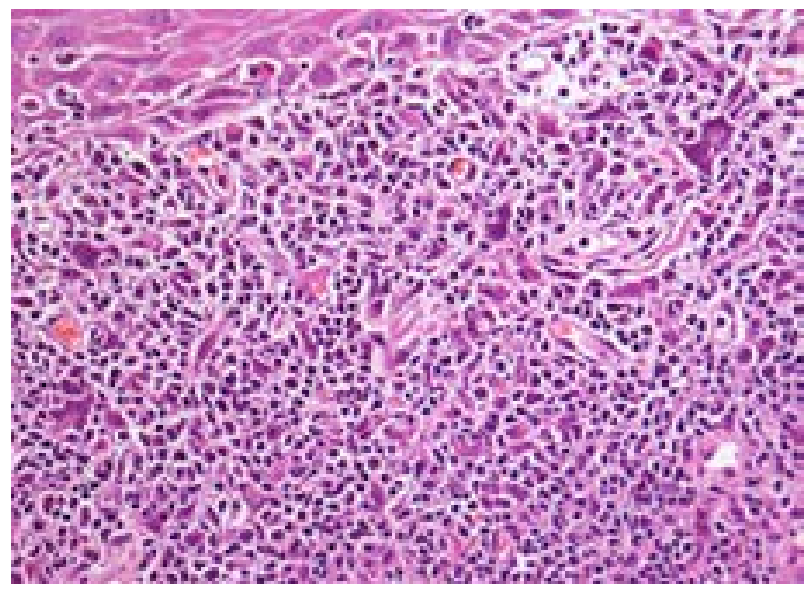

Figure 3. High-power H\&E-stained section demonstrating the subepithelial giant cells (original magnification x 200).

No evidence of granulomatous inflammation or polarisable foreign body material could be identified. No fungal 
elements or acid-fast bacilli were identified on special histochemical stains. A S100 immunohistochemical stain highlighted numerous dendritic cells, but was negative in the multinucleated giant cells. A CD68 immunohistochemical stain highlighted the multinucleated giant cells (Figure 4).

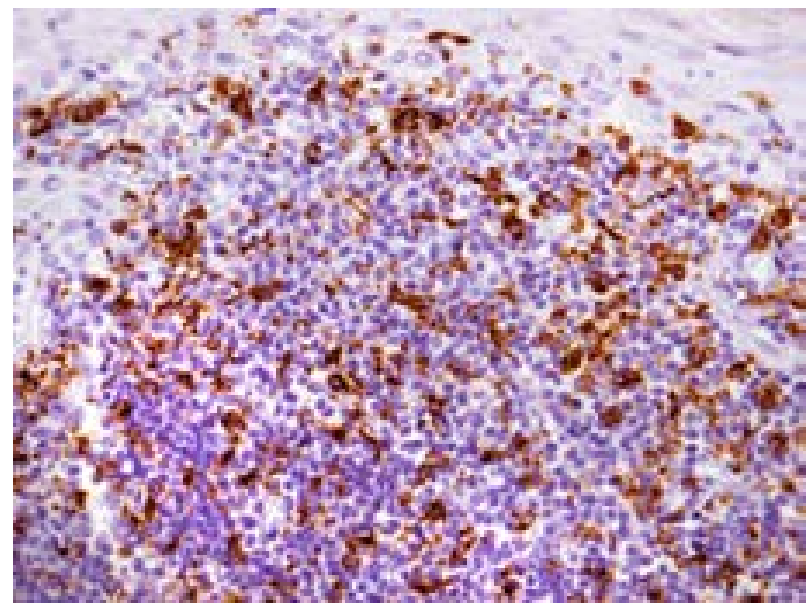

Figure 4. CD68 immunohistochemical stain confirming the histiocytic nature of the giant cells (original magnification $\times 100$ ).

An online literature search followed, whereby no reported cases of oral lichen planus or oral lichenoid lesions with multinucleated giant cells could be found. Several case reports of lichenoid granulomatous stomatitis with associated giant cells were referenced, however this entity was excluded in the current case due to the lack of granulomatous inflammation. A rare dermatological entity termed giant cell lichenoid dermatitis was mentioned in isolated papers, showing histological features similar to those described in the current case, most notably the presence of numerous multinucleated giant cells.

Following the literature search, the case was reported as giant cell lichenoid stomatitis, with a comment that the histological features mimic those of the dermatological entity, and may therefore be considered as an analogous mucosal entity.

\section{DISCUSSION}

Oral lichenoid lesions (OLLs), also termed oral lichenoid reactions (OLRs), are intraoral white lesions with a reticular, striated appearance, clinically similar to oral lichen planus. ${ }^{1}$ OLLs are considered an allergic response by the oral mucosa to dental materials, certain systemic medications, patients with graft-vs-host disease and patients with certain systemic diseases. ${ }^{2}$ OLLs represent a frequent oral condition, with a prevalence of $2.4 \%$ in the general population. They generally occur in adult patients with a mean age of 53-years and a female predominance. $^{2}$ The lesions are mostly present on the buccal mucosa, lateral border of the tongue and oral mucosa of the lips. Generally, the lesions are unilateral, an important feature distinguishing OLLs from oral lichen planus (OLP). Microscopically, OLLs and OLP show similar histological features, and cannot be easily differentiated. ${ }^{1-2} \mathrm{~A}$ band-like inflammatory cell infiltrate subjacent to the basal cells with associated basal cell vacuolar degeneration and apoptotic keratinocytes are features shared by both conditions. Subtle differences between the two entities include a more diffuse, mixed inflammatory infiltrate with a perivascular distribution seen in OLLs. ${ }^{3}$ Other common conditions with histological features mimicking OLP/OLLs include lupus erythematosus, chronic graft-vs-host disease and oral dysplasia. ${ }^{1,3}$ The malignant potential of OLP/OLLs is challenging due to their overlapping histological features, and the presence of a "lichenoid" inflammatory infiltrate commonly seen in dysplastic lesions. However, two recent systematic reviews showed that OLP had a malignant transformation rate of $1.09 \%$ and $0.9 \%$, whereas the rates for OLLs were $3.2 \%$ and $2.5 \%$, respectively. ${ }^{4}$

Lichenoid granulomatous stomatitis (LGS) was first described in 2006 by Robinson et al. Histologically, LGS consists of three distinctive components. First, is the presence of lichenoid inflammation, characterised by hyperkeratosis, basal cell degeneration with associated apoptotic bodies and a band-like lymphohistiocytic inflammatory cell infiltrate. Secondly, variable degrees of granulomatous inflammation can be seen in the lamina propria consisting of epithelioid macrophages without giant cells or areas of necrosis. Thirdly, lymphoid follicles are present showing a prominent perineural distribution. ${ }^{5}$ As previously mentioned, this condition was excluded in the current case due to a lack of granulomatous inflammation.

The term giant cell lichenoid dermatitis (GCLD) was first coined by Gonzalez et al. in 1986 as an unusual drug reaction. ${ }^{6}$ The patient in this original case report was a 52-year-old female who presented with a generalised pruritic eruption involving the trunk and extremities, with sparing of the palms, soles, face and mucous membranes. At the time of presentation, the patient was taking prednisone for systemic lupus erythematosus (SLE) and methyldopa and chlorothiazide for hypertension. Microscopic examination of skin punch biopsies showed a band-like chronic inflammatory cell infiltrate in the papillary dermis consisting of lymphocytes, histiocytes, and occasional plasma cells. A striking feature was the presence of numerous multinucleated giant cells. The epidermis showed alternating areas of atrophy and acanthosis with focal areas of vacuolar degeneration. Drug intake as an aetiological agent was supported by the fact that there was clinical improvement following discontinuation of the chronic medications the patient was taking. ${ }^{6-7}$ In this original case report, the authors postulated that the cause of the giant cells might be related to an impaired immune system due to longstanding SLE and low-dose corticosteroid administration.

Goldberg et al. further expanded on the condition with a report of three additional cases of GCLD. ${ }^{8}$ Notably, all three patients were in their $6^{\text {th }}-7^{\text {th }}$ decades of life and reported a history of chronic medication usage. Their cases differed slightly from the original report, as only one case demonstrated eosinophils in the inflammatory infiltrate. One of the patients in this series developed sarcoidosis, prompting the authors to examine these two entities in further detail. Both GCLD and sarcoidosis of the skin show similar clinical and histological features. ${ }^{7}$ Although cutaneous lesions are rare in sarcoidosis, they generally present with purple to 
brown papules and plaques, however, cases presenting with a pruritic scaly rash have also been described. ${ }^{8}$ Histologically, sarcoidosis of the skin is characterised by so-called naked granulomas containing multiple multinucleated giant cells in a lichenoid pattern at the dermo-epidermal junction. These features show some degree of overlap with GCLD, however GCLD lacks the characteristic granulomatous inflammatory component. ${ }^{7}$ The importance of considering sarcoidosis as a possible diagnosis for GCLD lies in its association with systemic disease. The authors concluded by emphasising the importance of a clinical work-up to exclude sarcoidosis in all patients with clinical and histological features of GCLD. 8

GCLD has also been reported in a patient with Baboon syndrome (symmetrical drug-related intertriginous and flexural exanthema) three days after administration of intravenous amoxicillin-clavulanic acid. ${ }^{9}$ Cordoba et al. described a patient with acute lymphoblastic leukaemia who developed GCLD limited to the site of scar formation following herpes zoster resolution. ${ }^{10}$ In both case reports, authors suggested that a hypersensitivity reaction could have played a role in triggering the condition, supporting the view of Gonzalez et al. , $9-10^{-10}$

\section{CONCLUSION}

In summary, the current case showed histological features of a mixed lichenoid inflammatory infiltrate with multinucleated giant cells, in the absence of infective aetiological agents and without evidence of granulomatous inflammation, and is therefore best termed giant cell lichenoid stomatitis. It is proposed that this entity should be considered analogous to a rare dermatological condition, giant cell lichenoid dermatitis, which shows similar histological features. Although the patient reported no history of medication usage, the concurrent psoriasis provides possible evidence of a dysregulated immune system that could account for the development of the current mucosal lesions. Unfortunately, due to limited reported cases, the clinical relevance and treatment implications of this entity are currently unclear.

\section{References}

1. Warnakulasuriya S. Clinical features and presentation of oral potentially malignant disorders. Oral Surg Oral Med Oral Pathol Oral Radiol. 2018; 125(6): 582-90.

2. Grossmann SDMC. Oral lichenoid lesion: A review of the literature. World Journal of Stomatology. 2015; 4(2): 103.

3. Muller S. Oral lichenoid lesions: Distinguishing the benign from the deadly. Mod Pathol. 2017; 30(s1): S54-S67.

4. Speight PM, Khurram SA, Kujan O. Oral potentially malignant disorders: Risk of progression to malignancy. Oral Surg Oral Med Oral Pathol Oral Radiol. 2018; 125(6): 612-27.

5. Robinson CM, Oxley JD, Weir J, Eveson JW. Lichenoid and granulomatous stomatitis: An entity or a non-specific inflammatory process? J Oral Pathol Med. 2006; 35(5): 262-7.

6. Gonzalez JG, Marcus MD, Cruz DJ. Giant cell lichenoid dermatitis. J Am Acad Dermatol. 1986; 15(1): 87-92.

7. Khullar G, De D, Saikia UN, Handa S. An unusual case of idiopathic localized giant cell lichenoid dermatitis. J Cutan Pathol. 2017; 44(8): 722-3.

8. Goldberg LJ, Goldberg N, Abrahams I, Silvers DN, Szaniawski W, Halperin AJ. Giant cell lichenoid dermatitis: A possible manifestation of sarcoidosis. J Cutan Pathol. 1994; 21(1): 47-51.

9. Khelifa-Hamdani E, Touati-Serraj M, Perriard J, Chavaz P, Saurat JH, Kaya G. Giant cell lichenoid dermatitis in a patient with Baboon syndrome. J Cutan Pathol. 2008; 35 Suppl. 1: 17-9.

10. Cordoba S, Fraga J, Bartolome B, Garcia-Diez A, Fernandez-Herrera J. Giant cell lichenoid dermatitis within herpes zoster scars in a bone marrow recipient. J Cutan Pathol. 2000; 27(5): 255-7. 